\title{
PREVISÃo AGROMETEOROLÓGICA DA DATA DE COLHEITA PARA A VIDEIRA 'NIAGARA ROSADA' $\left({ }^{1}\right)$
}

\author{
MÁRIO JOSÉ PEDRO JÚNIOR $\left({ }^{2,4}\right)$, PAULO CESAR SENTELHAS $\left({ }^{2}\right)$ \\ e FERNANDO PICARELLI MARTINS $\left({ }^{3}\right)$
}

\begin{abstract}
RESUMO
Mediante dados fenológicos da videira 'Niagara Rosada' observados na região de Jundiaí (SP), entre 1986 e 1993, foram avaliados três métodos agrometeorológicos para previsão da data provável de colheita, a saber: (a) data do florescimento mais 85 dias; (b) data do acúmulo de 1.000 graus-dia (temperatura-base $=10^{\circ} \mathrm{C}$ ) a partir da data da poda mais 42 dias; (c) data do acúmulo de 1.200 graus-dia a partir da data da poda mais 26 dias. Os métodos que utilizaram graus-dia apresentaram as melhores estimativas, com erros de 4 dias em relação à data observada de colheita, recomendando-se, então, o método $b$, por permitir previsōes com antecedência de 42 dias.
\end{abstract}

Termos de indexação: videira, 'Niagara Rosada', data de colheita, métodos agrometeorológicos.

\section{ABSTRACT \\ AGROMETEOROLOGICAL FORECAST OF HARVESTING DATE FOR 'NIAGARA ROSADA' GRAPE CROP}

Phenological data of the grape 'Niagara Rosada' obtained at Jundiaí, São Paulo State, Brazil, from 1986 to 1993, was used to evaluate three agrometeorological methods to predict the probable harvesting date: a) flowering date plus 85 days; b) date of accumulation of 1,000 degree-days (base temperature $=10^{\circ} \mathrm{C}$ ) from pruning date plus 42 days; c) date of accumulation of 1,200 degree-days from pruning date plus 26 days. The methods that utilized degree-days showed the best estimates with errors of 4 days in relation to observed harvesting dates, then, it is recommended the use of the $\mathbf{b}$ method by allowing forecasts 42 days in advance.

Index terms: grape, 'Niagara Rosada', harvesting date, agrometeorological methods.

(1) Recebido para publicação em 23 de novembro de 1993 e aceito em 26 de abril de 1994.

( ${ }^{2}$ Seção de Climatologia Agrícola, Instituto Agronômico (IAC), Caixa Postal 28, 13001-970 Campinas (SP).

( ${ }^{3}$ Estação Experimental de Jundiaí, IAC.

(4) Com bolsa de pesquisa do CNPq. 


\section{INTRODUÇÃO}

A previsão da época de maturação ou de colheita da videira é de interesse tanto do produtor, para planejamento de sua colheita, quanto dos órgãos governamentais, para previsão de safra e comercialização. O início da colheita comercial da videira varia muito de ano para ano, e sua determinação antecipada se torna importante para os produtores e comerciantes programarem suas atividades (Brink, 1974). Entre os fatores que influenciam a duração do ciclo da videira, desde a poda até à colheita, a temperatura do ar mostrou ser um dos principais, como constatado por Pedro Júnior et al. $\left({ }^{5}\right)$ para a videira 'Niagara Rosada' na região de Jundiaí (SP), quando determinaram os índices biometeorológicos para a cultura.

Os métodos de previsão da época de maturação da videira mais utilizados baseiam-se em: número médio de dias após o florescimento (Morris et al., 1980a); temperatura do ar através do acúmulo de graus-dia (Brink, 1974) e análise da concentração de ácido málico (Maujean et al., 1983, e Iacono et al., 1985).

Os métodos mais simples que utilizam a data de florescimento ou a temperatura do ar têm-se mostrado bastante eficazes na previsão da data de colheita, apresentando pequenos erros, respectivamente, 11 e 3 dias, e permitindo planejamentos com antecedência de aproximadamente 3 meses (Morris et al., 1980b; Brink, 1974), quando comparados aos que usam técnicas mais complexas, como a da concentração de ácido málico, com erros de 3 dias, porém com antecedência de 17 dias (Maujean et al., 1983).

Para avaliar a possibilidade de emprego dessas técnicas, foi desenvolvido este trabalho para testar e/ou adaptar métodos de previsão da data provável de colheita da uva 'Niagara Rosada', para a região produtora de Jundiaí (SP), com base na data de florescimento e/ou no acúmulo de graus-dia.

( 5 ) PEDRO JÚNIOR, M.J.; SENTELHAS, P.C.; POMMER, C.V. \& MARTINS, F. P. Graus-dia e índice biometeorológico para a videira 'Niagara rosada'. Revista Brasileira de Agrometeorologia, Santa Maria. (No prelo)

\section{MATERIAL E MÉTODO}

O experimento foi realizado na Estação Experimental de Jundiaí, do Instituto Agronômico de Campinas (lat.: $23^{\circ} 12^{\prime} \mathrm{S}$; long.: $46^{\circ} 53^{\prime} \mathrm{W}$; alt.: 715 m.), nos anos agrícolas de 1986/87 a $1992 / 93 \mathrm{e} e m$ vinhedos comerciais no Bairro da Toca e do Bom Jardim, no município de Jundiaí, nos anos agrícolas de 1991/92 e 1992/93.

A videira 'Niagara Rosada' foi conduzida em espaldeira com três fios de arame e espaçamento de $2 \times 1 \mathrm{~m}$. As datas da poda variaram de ano para ano, sendo realizadas em: 1\%/7, 15/7, 1\%/8, $15 / 8,1 \%$ e $15 / 9$.

Foram observados semanalmente os estádios fenológicos por uma escala de notas ( 1 a 17), adaptada por Pedro Júnior et al. (1990). As temperaturas do ar, máxima e mínima, foram observadas diariamente por termógrafos instalados nas áreas experimentais.

Utilizaram-se os dados fenológicos e de temperatura do ar para o desenvolvimento de métodos baseados nos de MORRIS et al. (1980a), para a data de florescimento, e por Brink (1974), para graus-dia (GD).

A partir dos dados obtidos na E.E. de Jundiaí, durante os anos agrícolas de 1986/87 a 1990/91, foram determinados: o número médio de dias entre a data do florescimento e a data de colheita; o número de dias entre a data de acúmulo de 1.000 e 1.200 GD, a partir da data da poda e a data da colheita, utilizando-se a temperatura-base de $10^{\circ} \mathrm{C}\left({ }^{5}\right)$. Para validação dos métodos, adotaram-se os dados fenológicos obtidos durante os anos agrícolas de 1991/92 e 1992/93 em três locais topoclimaticamente diferentes: Estação Experimental de Jundiaí (no local do ensaio), e nos bairros da Toca e Bom Jardim, onde foram, também, observados os estádios fenológicos da cultura.

Os métodos de previsão da data de colheita foram testados por comparação entre os dados observados e estimados através de análise de regressão linear, para verificar a precisão, e do índice de concordância de Willmott (índice d), 
apresentado por Willmott et al. (1985), para verificar a exatidão. O índice d de Willmott é obtido pela seguinte equação:

$$
\mathrm{d}=1-\left[\sum(\mathrm{Pi}-\mathrm{Oi})^{2} / \sum(|\mathrm{Pi}-\mathrm{O}|+|\mathrm{Oi}-\mathrm{O}|)^{2}\right]
$$

onde:

Pi é o valor previsto; Oi, o valor observado e $\mathrm{O}$, a média dos valores observados.

\section{RESULTADOS E DISCUSSÃO}

Primeiramente, determinaram-se os números de dias entre a data de florescimento e a data da colheita, e entre a data de acúmulo de $1.000 \mathrm{e}$ $1.200 \mathrm{GD}$ e a data da colheita, com dados da E.E. de Jundiaí nos anos agrícolas 1986/87 a 1990/91 (Quadro 1). Os valores médios obtidos são os seguintes: (a) Data de florescimento +85 dias; (b) Data de acúmulo de $1.000 \mathrm{GD}+42$ dias, e (c) Data de acúmulo de $1.200 \mathrm{GD}+26$ dias

Quadro 1. Número de dias necessários para atingir a colheita após a data de florescimento e de acúmulo de 1.000 e 1.200 graus-dia, para a videira 'Niagara Rosada', em Jundiaí (SP)

\begin{tabular}{|c|c|c|c|c|c|c|c|c|}
\hline \multirow{2}{*}{ Ano } & \multirow{2}{*}{$\operatorname{Poda}\left({ }^{1}\right)$} & \multirow{2}{*}{ Colheita $\left({ }^{2}\right)$} & \multicolumn{2}{|c|}{ Florescimento } & \multicolumn{2}{|c|}{$1.000 \mathrm{GD}$} & \multicolumn{2}{|c|}{$1.200 \mathrm{GD}$} \\
\hline & & & $\operatorname{Data}\left({ }^{3}\right)$ & $\operatorname{Dias}\left({ }^{4}\right)$ & $\operatorname{Data}\left({ }^{3}\right)$ & $\operatorname{Dias}\left({ }^{4}\right)$ & $\operatorname{Data}\left({ }^{3}\right)$ & $\operatorname{Dias}\left({ }^{4}\right)$ \\
\hline \multirow[t]{5}{*}{86} & $15 / 07$ & $11 / 12$ & $16 / 09$ & 86 & $30 / 10$ & 42 & $16 / 11$ & 25 \\
\hline & $1 \% 08$ & $11 / 12$ & $25 / 09$ & 77 & $07 / 11$ & 34 & $25 / 11$ & 16 \\
\hline & $15 / 08$ & $23 / 12$ & $04 / 10$ & 80 & $20 / 11$ & 33 & $04 / 12$ & 19 \\
\hline & $1 \% / 09$ & $06 / 01$ & $16 / 10$ & 82 & $29 / 11$ & 38 & $14 / 12$ & 22 \\
\hline & $15 / 09$ & $13 / 01$ & $24 / 10$ & 81 & $08 / 12$ & 36 & $23 / 12$ & 24 \\
\hline \multirow[t]{5}{*}{87} & $15 / 07$ & $04 / 12$ & $09 / 09$ & 86 & $28 / 10$ & 37 & $13 / 11$ & 21 \\
\hline & $1 \% / 08$ & $17 / 12$ & $22 / 09$ & 86 & $10 / 11$ & 37 & $26 / 11$ & 21 \\
\hline & $15 / 08$ & $28 / 12$ & $02 / 10$ & 87 & $18 / 11$ & 40 & $03 / 12$ & 25 \\
\hline & $1 \% / 09$ & $04 / 01$ & $15 / 10$ & 81 & $29 / 11$ & 36 & $14 / 12$ & 19 \\
\hline & $15 / 09$ & $11 / 01$ & $26 / 10$ & 77 & $08 / 12$ & 34 & $23 / 12$ & 19 \\
\hline \multirow[t]{5}{*}{88} & $15 / 07$ & $16 / 12$ & $13 / 09$ & 94 & $30 / 10$ & 47 & $18 / 11$ & 28 \\
\hline & $1 \% / 08$ & $23 / 12$ & $22 / 09$ & 92 & $08 / 11$ & 45 & $24 / 11$ & 29 \\
\hline & $15 / 08$ & $28 / 12$ & $30 / 09$ & 89 & $16 / 11$ & 42 & $04 / 12$ & 24 \\
\hline & $1 \% / 09$ & $10 / 01$ & $11 / 10$ & 91 & $1 \% 12$ & 40 & $17 / 12$ & 24 \\
\hline & $15 / 09$ & $17 / 01$ & $29 / 10$ & 80 & $13 / 12$ & 35 & $28 / 12$ & 20 \\
\hline \multirow[t]{5}{*}{89} & $15 / 07$ & $29 / 12$ & $07 / 10$ & 83 & $04 / 11$ & 55 & $22 / 11$ & 37 \\
\hline & $1 \% / 08$ & $04 / 01$ & $04 / 10$ & 92 & $15 / 11$ & 50 & $1 \% 12$ & 34 \\
\hline & $15 / 08$ & $11 / 01$ & $11 / 10$ & 92 & $22 / 11$ & 50 & $08 / 12$ & 34 \\
\hline & $1 \% / 09$ & $18 / 01$ & $24 / 10$ & 86 & $05 / 12$ & 44 & $20 / 12$ & 29 \\
\hline & $15 / 09$ & $25 / 01$ & $1 \% 11$ & 85 & $15 / 12$ & 41 & $1 \% 01$ & 24 \\
\hline \multirow[t]{5}{*}{90} & $15 / 07$ & $13 / 12$ & $18 / 09$ & 85 & $31 / 10$ & 43 & $14 / 11$ & 29 \\
\hline & $1 \% 08$ & $19 / 12$ & $27 / 09$ & 83 & $08 / 11$ & 41 & $21 / 11$ & 28 \\
\hline & $15 / 08$ & $03 / 01$ & $07 / 10$ & 88 & $15 / 11$ & 49 & $28 / 11$ & 36 \\
\hline & $1 \% / 09$ & $09 / 01$ & $17 / 10$ & 84 & $24 / 11$ & 46 & $07 / 12$ & 33 \\
\hline & $15 / 09$ & $18 / 01$ & $26 / 10$ & 84 & $02 / 12$ & 47 & $17 / 12$ & 32 \\
\hline \multicolumn{3}{|c|}{ Média } & & 85 & & 42 & & 26 \\
\hline \multicolumn{3}{|c|}{ Desvio padrão } & & 4,7 & & 5,9 & & 5,9 \\
\hline \multicolumn{2}{|c|}{$\mathrm{CV}(\%)$} & & & 6 & & 14 & & 23 \\
\hline
\end{tabular}

(1) Data da poda. $\left({ }^{2}\right)$ Data de observação da colheita. $\left({ }^{3}\right)$ Data de observação. $\left({ }^{4}\right)$ Dias até a colheita. 
O teste das estimativas da época de colheita pelos três métodos, foi feito com os dados obtidos durante os anos agrícolas de $1991 / 92$ e 1992/93, para quatro épocas de poda para os três locais do experimento: E.E. de Jundiaí, Bairro da Toca e Bairro Bom Jardim
(Quadro 2), permitindo verificar que os métodos que utilizaram os graus-dia tiveram erros bem inferiores ao que empregou a data de florescimento, fato também constatado por Brink (1974), nos Estados Unidos, para a videira 'Concord'.

Quadro 2. Data da poda e colheita da uva 'Niagara Rosada' observada e estimada pela data de florescimento + 85 dias e pelo acúmulo de $1.000 \mathrm{GD}+42$ dias e $1.200 \mathrm{GD}+26$ dias para diferentes locais da região produtora de Jundiaí (SP)

\begin{tabular}{|c|c|c|c|c|c|c|c|c|c|}
\hline \multirow{2}{*}{ Local $\left({ }^{1}\right)$} & \multirow{2}{*}{ Ano } & \multirow{2}{*}{ Poda $\left({ }^{2}\right)$} & \multirow{2}{*}{ Colheita $\left({ }^{3}\right)$} & \multicolumn{2}{|c|}{ Flor. + 85 dias } & \multicolumn{2}{|c|}{$1.000 \mathrm{GD}+42 \mathrm{dias}$} & \multicolumn{2}{|c|}{$1.200 \mathrm{GD}+26 \mathrm{dias}$} \\
\hline & & & & $\operatorname{Data}\left({ }^{4}\right)$ & $\operatorname{Dias}\left({ }^{5}\right)$ & $\operatorname{Data}\left({ }^{4}\right)$ & $\operatorname{Dias}\left({ }^{5)}\right.$ & $\operatorname{Data}\left({ }^{4}\right)$ & $\operatorname{Dias}\left({ }^{5}\right)$ \\
\hline \multirow[t]{8}{*}{ EEJ } & $91 / 92$ & $1 \% / 07$ & $23 / 12$ & $29 / 11$ & -24 & $24 / 12$ & 1 & $23 / 12$ & 0 \\
\hline & & $15 / 07$ & $23 / 12$ & $02 / 12$ & -21 & $29 / 12$ & 6 & $28 / 12$ & 5 \\
\hline & & $1: / 08$ & $07 / 01$ & $17 / 12$ & -21 & $05 / 01$ & -2 & $03 / 01$ & -4 \\
\hline & & $15 / 08$ & $14 / 01$ & $28 / 12$ & -17 & $10 / 01$ & -4 & $08 / 01$ & -6 \\
\hline & $92 / 93$ & $1 \% / 08$ & $30 / 12$ & $12 / 01$ & 13 & $26 / 12$ & -4 & $29 / 12$ & -1 \\
\hline & & $15 / 08$ & $06 / 01$ & $18 / 01$ & 12 & $04 / 01$ & -2 & $06 / 01$ & 0 \\
\hline & & $1 \% 09$ & $16 / 01$ & $27 / 01$ & 11 & $18 / 01$ & 2 & $17 / 01$ & 1 \\
\hline & & $15 / 09$ & $30 / 01$ & $14 / 02$ & 15 & $27 / 01$ & -3 & $26 / 01$ & -4 \\
\hline \multirow[t]{8}{*}{ TO } & $91 / 92$ & $1 \% / 07$ & $23 / 12$ & $29 / 11$ & -24 & $18 / 12$ & -5 & $19 / 12$ & -4 \\
\hline & & $15 / 07$ & $30 / 12$ & $05 / 12$ & -25 & $24 / 12$ & -6 & $24 / 12$ & -6 \\
\hline & & $1 \% / 08$ & $07 / 01$ & $18 / 12$ & -20 & $02 / 01$ & -5 & $03 / 01$ & -4 \\
\hline & & $15 / 08$ & $14 / 01$ & $25 / 12$ & -20 & $08 / 01$ & -6 & $08 / 01$ & -6 \\
\hline & $92 / 93$ & $1 \% / 08$ & $30 / 12$ & $12 / 01$ & 13 & $30 / 12$ & 0 & $02 / 01$ & 3 \\
\hline & & $15 / 08$ & $06 / 01$ & $11 / 01$ & 5 & $08 / 01$ & 2 & $11 / 01$ & 3 \\
\hline & & $1 \% / 09$ & $13 / 01$ & $19 / 01$ & 6 & $21 / 01$ & 8 & $21 / 01$ & 8 \\
\hline & & $15 / 09$ & $26 / 01$ & $20 / 01$ & -6 & $30 / 01$ & 4 & $29 / 01$ & 3 \\
\hline \multirow[t]{8}{*}{$\mathrm{BJ}$} & $91 / 92$ & $1 \% / 07$ & $10 / 12$ & $29 / 11$ & -11 & $03 / 12$ & -7 & $03 / 12$ & -7 \\
\hline & & $15 / 07$ & $17 / 12$ & $03 / 12$ & -14 & $10 / 12$ & -7 & $10 / 12$ & -7 \\
\hline & & $1 \% / 08$ & $24 / 12$ & $18 / 12$ & -6 & $20 / 12$ & -4 & $20 / 12$ & -4 \\
\hline & & $15 / 08$ & $31 / 12$ & $23 / 12$ & -8 & $28 / 12$ & -3 & $28 / 12$ & -3 \\
\hline & $92 / 93$ & $1 \% 08$ & $30 / 12$ & $12 / 01$ & 13 & $27 / 12$ & -3 & $28 / 12$ & -2 \\
\hline & & $15 / 08$ & $04 / 01$ & $07 / 01$ & 3 & $05 / 01$ & 1 & $06 / 01$ & 2 \\
\hline & & $1 \% / 09$ & $13 / 01$ & $15 / 01$ & 2 & $17 / 01$ & 4 & $16 / 01$ & 3 \\
\hline & & $15 / 09$ & $30 / 01$ & $22 / 01$ & -8 & $24 / 01$ & -6 & $23 / 01$ & -7 \\
\hline \multicolumn{4}{|c|}{ Erro absoluto médio } & & 13,4 & & 4,0 & & 4,0 \\
\hline
\end{tabular}

$\left({ }^{1}\right)$ EEJ: Estação Experimental de Jundiaí; TO: Bairro da Toca; BJ: Bairro Bom Jardim. $\left({ }^{2}\right)$ Data da poda. $\left({ }^{3}\right)$ Data da observação da colheita. $\left({ }^{4}\right)$ Data estimada. $\left({ }^{5}\right)$ Data estimada e observada. 

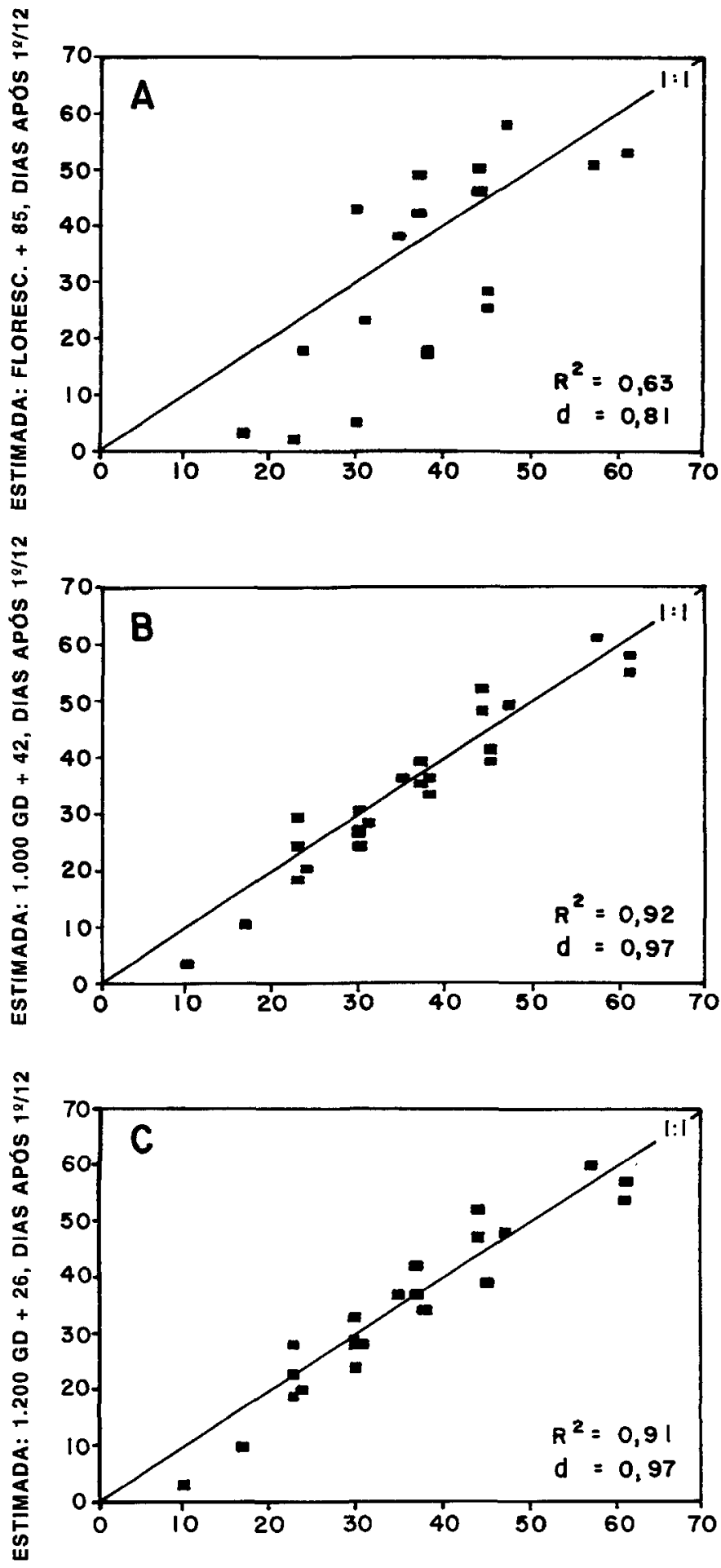

OBSERVADA, DIAS APÓS 1\%12

Figura 1. Comparação entre a data observada de colheita e a estimada pelos métodos A: data de florescimento mais 85 dias; B: data de acúmulo de 1.000 GD a partir da data da poda mais 42 dias; C: data de acúmulo de $1.200 \mathrm{GD}$ a partir da data da poda mais 26 dias, para diferentes locais da região produtora de Jundiaí (SP). 
Quadro 3. Análise estatística dos métodos de estimativa da data de colheita da uva 'Niagara

Rosada' para a região produtora de Jundiaí (SP)

\begin{tabular}{lccccrr}
\hline Método de estimativa & $\mathbf{R}^{2}$ & Índice $d$ & Es & Ea & RQE & EAM \\
\hline Floresc. +85 dias & 0,63 & 0,81 & 7,06 & 13,30 & 15,06 & 13,4 \\
1.000 GD +42 dias & 0,92 & 0,97 & 2,00 & 4,07 & 4,53 & 4,0 \\
1.200 GD +26 dias & 0,91 & 0,97 & 1,56 & 4,27 & 4,55 & 4,0 \\
\hline
\end{tabular}

Obs.- $\mathrm{R}^{2}$ : coeficiente de determinação (precisão); índice d: coeficiente de exatidão; Es: erro sistemático; Ea: erro aleatório; RQE: raiz quadrada do erro; EAM: erro absoluto médio.

Quadro 4. Condições térmicas médias para os anos agrícolas 1991/92 e 1992/93 e normais para o período do ano correspondente ao ciclo da videira 'Niagara Rosada' na Estação Experimental de Jundiaí (SP)

\begin{tabular}{|c|c|c|c|}
\hline \multirow{2}{*}{ Mês } & \multicolumn{3}{|c|}{ Temperatura média mensal $\left({ }^{\circ} \mathrm{C}\right)$} \\
\hline & $1991 / 92$ & $1992 / 93$ & Normal \\
\hline Julho & 17,1 & 17,8 & 17,2 \\
\hline Agosto & 19,2 & 18,3 & 18,6 \\
\hline Setembro & 20,2 & 19,4 & 19,8 \\
\hline Outubro & 21,6 & 21,8 & 21,4 \\
\hline Novembro & 23,2 & 22,3 & 22,3 \\
\hline Dezembro & 24,0 & 22,4 & 23,0 \\
\hline Janeiro & 23,7 & 24,1 & 23,6 \\
\hline Média & 21,3 & 20,8 & 20,8 \\
\hline
\end{tabular}

O erro absoluto médio na estimativa da data de colheita dos métodos que usaram os graus-dia foram, aproximadamente, de 4 dias, diferindo significativamente do erro absoluto médio do modelo que empregou o florescimento, que foi superior a 13 dias. Esse resultado é bastante próximo do encontrado por Morris et al. (1980b): de 11 dias. Além disso, pode-se verificar - Quadro 3 e Figura 1 - que a precisão $\left(\mathrm{R}^{2}\right)$ e a exatidão (índice $\mathrm{d}$ ) dos métodos que utilizaram graus-dia foram maiores que as daquele que utilizou a data do florescimento, cujo erro sistemático foi bastante elevado.
No quadro 2, pode-se verificar que o erro absoluto médio foi sistematicamente menor em $1992 / 93$ do que em 1991/92. Isso pode ser explicado pela ocorrência de temperaturas mais elevadas no ano agrícola de 1991/92, principalmente durante os meses de novembro e dezembro (Quadro 4), que contribuíram para antecipar a colheita. Como os métodos usaram o critério de número médio de dias após: florescimento e acúmulo de 1.000 e de 1.200 GD; quanto maior seu valor, maior poderá ser o erro absoluto médio da estimativa da data de colheita, pois o efeito da temperatura não estará sendo considerado.

\section{CONCLUSÕES}

1. Entre os dois métodos que utilizaram os graus-dia não houve diferença, pois os valores dos índices de precisão e exatidão e dos erros das estimativas são praticamente iguais.

2. Pode-se adotar, para previsão da data provável de colheita da uva 'Niagara Rosada', o método que utiliza a data de acúmulo de 1.000 GD a partir da data da poda, porque permite previsões da data de colheita com uma antecedência de 42 dias.

\section{REFERÊNCIAS BIBLIOGRÁFICAS}

BRINK, C. van den. Predicting harvest date of the 'Concord' grape crop in Southwest Michigan. HortScience, Alexandria, 9(3):206-208, 1974. 
IACONO, F.; FREGONI, M. \& ZAMBONI, M. Evoluzione della maturazione e previsione della data più probabile de vendemmia. Vignevini, Bologna, 12(9):7-12, 1985.

MAUJEAN, A.; BRUN, O.; VESSELlE, G.; BUREAU, G.; BOUCHER, J. M.; COUSIN, M. \& FEUILLAT, $M$. Étude de la maturation de cépages champenois - Modèles de prévision de la date de vendange. Vitis, Siebeldingen, 22(2):137-150, 1983.

MORRIS, J.R; CAWTHON, D.L.; SPAYD, S.E.; MAY, R. D. \& BRYAN, D. R. Prediction of 'Concord' grape maturation and sources of error. Journal of the American Society for Horticultural Science, Alexandria, 105(3):313-318, 1980.
MORRIS, J.R; CAWTHON, D.L.; SPAYD, S.E.; MAY, R. D. \& BRYAN, D. R. Prediction of 'Concord' grape harvest. Arkansas Farm Research, Fayetteville, 29(3):11, 1980.

PEDRO JÚNIOR, M.J.; RIBEIRO, I.J.A.; POMMER, C.V. \& MARTINS, F. P. Caracterização de estádios fenológicos da videira 'Niagara rosada'. In: CONGRESSO BRASILEIRO DE FRUTICULTURA, 10., Fortaleza, 1990. Anais. Fortaleza, Sociedade Brasileira de Fruticultura, 1990. p.453-456.

WILLMOTT, C.J.; ACKLESON, S.G.; DAVIS, R.E.; FEDDEMA,J.J.; KLINK, K.M.; LEGATES, D.R.; O'DONNELL, J. \& ROWE, C.M. Statistics for the evaluation and comparison of models. Journal of Geophysical Research, Washington, D.C., 90(c5):8995-9005, 1985. 